\title{
Mobilização precoce para reabilitação de pacientes acometidos por COVID-19 em Unidade de Terapia Intensiva: revisão integrativa
}

\author{
Mobilization for the rehabilitation of patients affected by COVID-19 in the intensive care unit: an \\ integrative review \\ Movilización temprana para la rehabilitación de pacientes afectados por COVID-19 en la unidad \\ de cuidados intensivos: revisión integrativa
}

Recebido: 14/10/2021 | Revisado: 20/10/2021 | Aceito: 25/10/2021 | Publicado: 28/10/2021

\author{
Laura Maria Andrade Coelho \\ ORCID: https://orcid.org/0000-0002-0718-9542 \\ Centro Universitário do Norte, Brasil \\ E-mail: lauramariia98@gmail.com \\ Bárbara Lira Bahia Mendes \\ ORCID: https://orcid.org/0000-0002-8363-3211 \\ Centro Universitário do Norte, Brasil \\ E-mail: fisio.barbara@hotmail.com
}

\begin{abstract}
Resumo
Introdução: A mobilização precoce é um recurso da fisioterapia com abordagem multiprofissional que visa diminuir os efeitos físicos e psicológicos decorrentes da imobilidade durante o tempo de internação. Objetivo: analisar a importância das técnicas de mobilização precoce em pacientes acometidos pela COVID-19 internados em UTI, bem como as barreiras e dificuldades encontradas pelos profissionais para a realização dessa intervenção. Metodologia: Revisão integrativa realizada através da leitura documental de artigos encontrados nas bases de dados PubMED, BVS (Biblioteca Virtual em Saúde), SciELO (Brasil Scientific Electronic Library Online) e Google Acadêmico, utilizando os seguintes descritores indexados no DeCS (Descritores em Ciências da Saúde): Mobilização precoce; Unidade de Terapia Intensiva; Fisioterapia e SARS-CoV-2. Resultados e Discussões: Para compor a discussão dessa revisão, foram selecionados de acordo com os critérios de busca, 10 artigos que abordaram a mobilização precoce em pacientes acometidos por COVID-19. Os artigos mostram que a prática da mobilização precoce pode impactar diretamente na funcionalidade do paciente, contribuir para diminuição do tempo de internação e de ventilação mecânica invasiva. No entanto barreiras como sedação, instabilidade hemodinâmica e atualmente o contexto de pandemia dado pela COVID-19, tem se tornado um desafio a ser superado para a realização da mobilização precoce dentro da UTI. Conclusão: Apesar das evidências encontradas a respeito dos benefícios da mobilização precoce, ainda existem uma série de barreiras que impossibilitam a realização eficiente da intervenção, limitações não somente ligadas diretamente ao paciente, mas também relacionadas a estrutura, organização e a equipe atuante na Unidade de Terapia Intensiva.
\end{abstract}

Palavras-chave: Mobilização precoce; Unidade de Terapia Intensiva; Fisioterapia; SARS-CoV-2.

\begin{abstract}
Introduction: Early mobilization is a physiotherapy resource with a multi-professional approach that aims to decrease the physical and psychological effects that occur during long ICU treatment. Objective: Analyze the importance of the early mobilization techniques in patients in the intensive care unit affected by COVID-19 as well as the obstacles and hardships faced by the professionals who try to execute this type of intervention. Methods: Integrative review performed through a documental reading of articles found in the database of PubMed, VHL (Virtual health library), SciELO (Brazil Scientific Electronic Library Online), and Google Scholar, using the following keywords in the DeCS (Health science descriptors): Early mobilization; Intensive unit care; Physiotherapy and SARS-CoV-2. Results and discussion: To compose the discussion of this review, 10 articles that addressed early mobilization in patients affected by COVID-19 were selected according to the search criteria. The articles present that the use of early mobilization can directly impact the functionality of the patient, contribute to shortening the length of stay in hospital and decrease the use of invasive mechanical ventilation. However, obstacles such as sedation, hemodynamic instability, and the pandemic caused by COVID-19 have been a challenge to be overcome to execute the early mobilization in the ICU. Conclusion: Despite the evidence found about the benefits of early mobilization, there are still many barriers that prevent an efficient implementation of the intervention, limitations not only related to the patient but also related to the structure and organization of the team working in the ICU.
\end{abstract}

Keywords: Early mobilization; Intensive Care Unit; Physiotherapy; SARS-CoV-2. 


\begin{abstract}
Resumen
Introducción: La movilización precoz es un recurso de fisioterapia con enfoque multidisciplinario que tiene como objetivo reducir los efectos físicos y psicológicos de la inmovilidad durante la hospitalización. Objetivo: Analizar la importancia de las técnicas de movilización temprana en pacientes afectados por COVID-19 hospitalizados en la UCI, así como las barreras y dificultades que encuentran los profesionales para llevar a cabo esta intervención. Metodología: Revisión integrativa realizada mediante lectura documental de artículos encontrados en PubMED, BVS (Virtual Health Library), bases de datos SciELO. (Brasil Scientific Electronic Library Online) y Google Académico, utilizando los siguientes descriptores indizado en DeCS (Descriptores en Ciencias de la Salud): Movilización temprana; Unidad de terapia intensiva; Fisioterapia y SARS-CoV-2. Resultados y discusiones: Para componer la discusión de esta revisión, se seleccionaron 10 artículos que abordaron la movilización temprana en pacientes afectados por COVID-19 según los criterios de búsqueda. Los artículos muestran que la práctica de la movilización precoz puede impactar directamente en la funcionalidad del paciente, contribuyendo a la reducción de la estancia hospitalaria y la ventilación mecánica invasiva. Sin embargo, barreras como la sedación, la inestabilidad hemodinámica y actualmente el contexto pandémico que brinda el COVID-19, se han convertido en un desafío a superar para la realización de la movilización temprana dentro de la UCI. Conclusión: A pesar de la evidencia encontrada sobre los beneficios de la movilización temprana, aún, existen una serie de barreras que imposibilitan realizar la intervención de manera eficiente, limitaciones no solo directamente ligadas al paciente, sino también relacionadas con la estructura, organización y equipo que trabaja en la Unidad de Cuidados Intensiva.
\end{abstract}

Palabras clave: Movilización precoz; Unidad de Terapia Intesiva; Fisioterapia; SARS-CoV-2.

\title{
1. Introdução
}

Segundo Sales et al. (2020), a COVID-19, patologia decorrente do novo variante da família corona vírus denominado SARS-CoV-2, teve seu primeiro caso relatado em novembro de 2019 na China. O novo vírus tem um alto nível de letalidade e disseminação, tendo alcançado diversos países em poucos meses após o primeiro caso identificado. Sendo assim, a Organização Mundial de Saúde (OMS) declarou a pandemia em março de 2020. De acordo com a OMS, até o presente momento em que foi realizado esse estudo, houve 229.858.719 casos confirmados de COVID-19 no mundo inteiro. Ademais o vírus pode ser transmitido principalmente por contato com gotículas salivares, secreções e outros fluidos do corpo, e tem como principais sinais e sintomas a febre, tosse seca, queda da saturação de oxigênio (SPO2), cefaléia, e distúrbios gastrointestinais.

Silva et al. (2020) apontam que alguns pacientes diagnosticados com a COVID-19 podem ser assintomáticos, isto é, não apresentar nenhum sinal ou sintoma, o que colabora para a rápida disseminação do vírus. No entanto, outros pacientes podem evoluir para distúrbios respiratórios mais graves como a Síndrome do Desconforto Respiratório Agudo (SARA), podendo necessitar de tratamento em Unidade de Terapia Intensiva (UTI). Esses pacientes em sua maioria são portadores de outras doenças crônicas metabólicas, respiratórias, cardiovasculares, ou até mesmo outras condições clínicas, sendo classificados como grupo de risco.

Segundo Bonorino e Cani (2020), pacientes acometidos pela COVID-19 que desenvolvem a fase crítica da doença e necessitam de cuidados intensivos, podem ter maiores efeitos deletérios após alta hospitalar, o que interfere diretamente na qualidade de vida do paciente. De acordo com McWilliams et al. (2021), a sedação profunda e o uso de bloqueadores neuromusculares são fatores de risco que colaboram para o desenvolvimento da fraqueza muscular adquirida na UTI, ocorrendo uma perda muscular de $20 \%$ na primeira semana de doença crítica.

De acordo com Pissolato et al. (2018), a fraqueza muscular, hipotrofia, e o descondicionamento físico são condições adquiridas decorrentes da imobilização prolongada no leito. Essas condições interferem diretamente no tempo que o paciente permanecerá em ventilação mecânica, dificultando o desmame e sua reabilitação funcional após alta hospitalar.

Para Pinto et al. (2020) a mobilização precoce é um recurso da fisioterapia com abordagem multiprofissional, que visa diminuir os efeitos físicos e psicológicos decorrentes da imobilidade durante o tempo de internação. Essa abordagem inclui exercícios motores que colaboram para a recuperação funcional do paciente, podendo ser considerada uma reabilitação inicial atuando em conjunto com o sistema respiratório. Além de promover a manutenção da força muscular e da mobilidade articular. 
Convém salientar, ainda, que segundo Filho et al. (2020) os protocolos de mobilização precoce têm se mostrado colaborativos para aumentar a taxa de desmame e diminuir o tempo de internação em UTI.

Sendo assim, a mobilização precoce é recomendada pela OMS e por outras associações em pacientes acometidos pela COVID-19, e deve ser realizada assim que o paciente se encontrar estável clinicamente, sendo baseada em protocolos e diretrizes já evidenciados e utilizados em pacientes críticos internados em UTI (Cordeiro \& Sarmento, 2020).

No entanto, Martinez e Andrade (2020), ressaltam que as técnicas de mobilização precoce podem estar sendo negligenciadas dentro do contexto de pandemia. Isso tem acontecido devido ao grande aumento de hospitalizações de pacientes nas UTIs e a sobrecarga do trabalho de fisioterapeutas e outros profissionais intensivistas. Com isso, o manejo respiratório e a busca por sobrevivência tem sido prioridades, podendo gerar complicações prolongadas na alta de pacientes críticos incluindo atrofia e fraqueza muscular.

Aquim et al. (2019) ressalta a importância de toda a equipe multidisciplinar no manejo dos pacientes, tendo o fisioterapeuta domínio específico da prescrição de atividades. Portanto, se faz necessário o estudo de procedimentos terapêuticos em todas as fases da doença e de recursos que promovam um olhar de cuidado integral ao paciente acometido pela COVID-19, submetido a permanência de média/longa duração em UTIs.

Diante do exposto acima, essa pesquisa tem como objetivo principal analisar a importância das técnicas de mobilização precoce em pacientes acometidos pela COVID-19 internados em UTI, bem como as barreiras e dificuldades encontradas pelos profissionais para a realização dessa intervenção.

\section{Metodologia}

Trata-se de uma Revisão Integrativa da literatura realizada através da leitura documental de artigos científicos sobre o uso da mobilização precoce em pacientes internados em UTI, com ênfase em pacientes acometidos pela COVID-19.

Foi realizada uma análise descritiva com abordagem qualitativa de protocolos, técnicas, benefícios e barreiras encontradas a respeito da realização da mobilização precoce dentro da UTI. Utilizando as 6 etapas da revisão integrativa que são: Identificação do tema e seleção da questão de pesquisa; Estabelecimento de critérios de inclusão e exclusão; Identificação dos estudos pré selecionados e selecionados; Categorização dos estudos selecionados; Análise e interpretação dos resultados e apresentação da revisão do conhecimento.

Também foi definido os seguintes descritores indexados no DeCS (Descritores em Ciências da Saúde): Mobilização precoce; Unidade de Terapia Intensiva; Fisioterapia e SARS-COV2. Para combiná-los entre si, foi utilizado o operador booleano AND nas bases de dados PubMED, BVS (Biblioteca Virtual em Saúde), SciELO (Brasil Scientific Electronic Library Online) e Google Acadêmico onde foi feita a busca por artigos cientificos entre os meses de março a setembro de 2021.

Inicialmente foram selecionados 73 artigos no total de acordo com os critérios de inclusão que foram: artigos disponíveis nos idiomas português e inglês, artigos publicados entre os anos de 2016 e 2021. Após aplicar os critérios de exclusão que foram: monografias, teses, dissertações, resumos, artigos pagos, artigos incompletos, artigos de anos anteriores a 2016, e abordagem em pacientes pediátricos, restaram 17 artigos pré-selecionados através da leitura do tema, resumo e palavras-chaves.

Após a leitura na íntegra, restaram 10 artigos que foram selecionados e tabelados, incluindo a síntese dos objetivos, metodologias e conclusões de cada um deles. O fluxograma do processo de seleção de artigos está representado na Figura 1. 
Figura 1 - Fluxograma do processo de seleção de artigos.

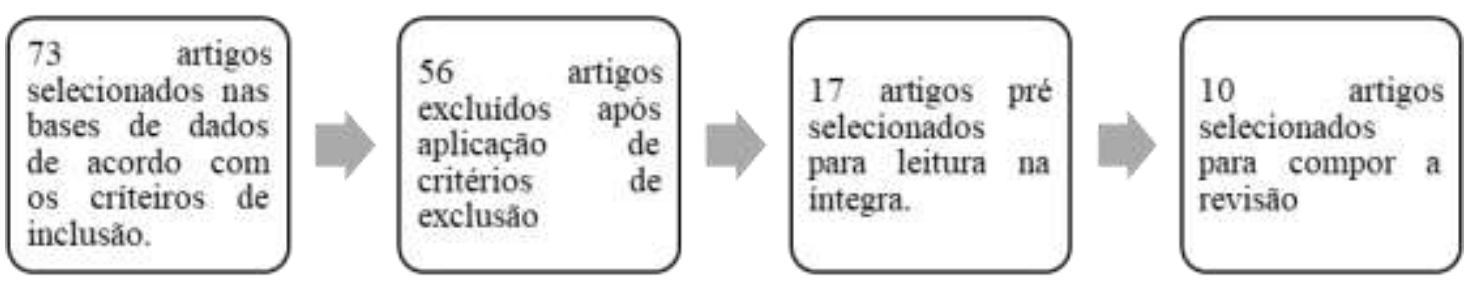

Fonte: Autores.

\section{Resultados e Discussão}

Para compor a discussão dessa revisão, foram selecionados, de acordo com os critérios de busca, 10 artigos que abordaram a mobilização precoce em pacientes acometidos por COVID-19 de acordo com a Tabela 1.

Tabela 1 - Resumo dos artigos selecionados.

\begin{tabular}{ll}
\hline AUTOR/ANO & \multicolumn{1}{c}{ OBJETIVO } \\
\hline \hline & $\begin{array}{l}\text { Determinar se há uma } \\
\text { correlação entre a mobilidade } \\
\text { funcional, } \\
\text { principalmente pela escala } \\
\text { PERME e o desfecho clínico } \\
\text { de pacientes internados em } \\
\text { uma UTI. }\end{array}$ \\
& \\
&
\end{tabular}

\section{METODOLOGIA}

Estudo prospectivo quantitativo longitudinal, realizado em um hospital Universitário em Fortaleza - Brasil, entre março e maio de 2017, com 33 indivíduos incluídos na amostra.

\section{CONCLUSÃO}

Houve uma relação entre o desfecho clínico dos pacientes e as pontuações de mobilidade, sendo baixas na escala PERME e altas na APACHE II, sendo associadas ao desfecho de morte. A presença de barreiras para a realização da mobilização precoce são fatores modificáveis, estando a baixa mobilidade associada a maior taxa de mortalidade.

\begin{tabular}{ll}
\hline \hline & \\
& \\
& Esclarecer o impacto físico da \\
& COVID-19 e a permanência \\
(McWilliams, et & em UTI e descrever a \\
al., 2021) & demografia, os níveis de \\
& reabilitação e o status de \\
mobilidade na alta da UTI.
\end{tabular}

\section{Estudo observacional realizado no Hospital Queen Elizabeth em Birmingham, na Inglaterra. 177 pacientes foram incluídos, internados em UTI em março e abril de 2020. Os dados foram coletados ao longo do período de avaliação usando anotações de pacientes e bancos de dados eletrônicos}

Apenas 110 pacientes sobreviveram na UTI e foram incluídos na análise. Todos os pacientes receberam um protocolo de mobilização na UTI, com início tardio devido as complicações da doença e tendo como uma das principais barreiras a sedação. Apesar disso, a mobilização era viável e metade dos pacientes que foram mobilizados tiveram a capacidade de se levantar e passar para uma cadeira antes da alta para enfermaria.
Estudo transversal com pacientes acometidos pela COVID-19 na fase pós aguda no hospital San Marco Polyclinic em Zingonia na Itália. 32 pacientes foram analisados os quais a maioria sofria de dispneia e falta de ar e estavam acamados.
Os achados nos pacientes podem fazer parte da síndrome pós terapia intensiva, considerada como uma possível complicação tardia de pacientes pós COVID-19. Esforços clínicos iniciais de médicos e fisioterapeutas são necessários para evitar essa condição. Sugeriu-se que as unidades de reabilitação deveriam constituir um estágio intermediário entre UTI e alta. reabilitação precoce de acordo com a função pulmonar. 
Avaliar se o protocolo de mobilização precoce contribui para a redução do tempo de

(Costa, et al., internação em Unidade de 2019) pacientes submetidos a ventilação mecânica invasiva e os efeitos na força muscular periférica.
Estudo de coorte

concorrente (prospectivo), realizado em uma UTI Adulto de um hospital público geral de médio porte da região do Vale do Sinos, RS, com pacientes adultos submetidos à VMI que estiveram internados nesta unidade de agosto a novembro de 2016.
O grupo de intervenção apresentou redução do tempo de VMI e tempo de internação na UTI e cerca de $83 \%$ dos pacientes do grupo de intervenção alcançaram o nível funcional 5 na alta da UTI, com capacidade para deambular. O protocolo de mobilização precoce é considerado um método seguro, viável e que não aumenta os custos hospitalares.
Reunir dados e evidências das (Silveira, et al., técnicas mais utilizadas na 2019) mobilização precoce de pacientes críticos.
Revisão sistemática onde foram incluídos 10 artigos em sua maioria ensaios randomizados e estudos experimentais, todos avaliados pela escala PEDro.
O ciclo ergômetro apresenta grandes benefícios quando utilizados na intervenção, e pode ser associado ou não a eletroestimulação. Técnicas tradicionais como a mobilização passiva e treinamento funcional a beira leito são mais utilizados na maioria dos estudos encontrados.

\begin{tabular}{ll}
\hline \hline & \\
& \\
& $\begin{array}{l}\text { Promover a confecção de um } \\
\text { instrumento, no formato de } \\
\text { Checklist avaliativo, } \\
\text { (Silva, et abordando os principais } \\
\text { pontos que um paciente crítico } \\
\text { deve apresentar em seu quadro } \\
\text { clínico, para ser eleito a } \\
\text { intervenção de mobilização } \\
\text { precoce. }\end{array}$
\end{tabular}
(Sakai, et al., eficiência da reabilitação na 2021) UTI e enfermaria para pacientes com COVID-19.

Abordagem do tipo
experimental aplicada
durante o acompanhamento
de 24 pacientes internados
na UTI do MPHU, no
município de Uberaba-
MG, no período de janeiro
a março de 2020, em duas
situações: beira leito e via
sistema de registro de
informações online, por
meio do acesso a um
programa com as a
avaliações dos pacientes.

Abordagem do tipo de 24 p companhamento na UTI do MPHU, no município de UberabaMG, no período de janeiro a março de 2020, em duas situações: beira leito e via sistema de registro de informações online, por programa com as avaliações dos pacientes.

A avaliação dos pacientes bem como dos critérios de segurança realizada antes da aplicação de mobilização precoce é essencial para o estabelecimento de um protocolo de atividades. A utilização do modelo checklist para essa avaliação pode favorecer a escolha da melhor conduta individual e diminuir as intercorrências.

Estudo observacional em um centro único (Tokyo Medical and Dental University) entre 21 de abril e 20 de agosto de 2020. Foram admitidos 161 pacientes com COVID-19, os quais 95 foram submetidos a terapia de reabilitação, sendo 78 diagnosticados com COVID moderado em alas de enfermaria e $17 \mathrm{com}$ COVID grave na UTI.
Na reabilitação em alas de enfermaria, 9,8\% dos pacientes receberam a reabilitação de forma remota, quando atendiam aos critérios de inclusão, e foram monitorados pelo fisioterapeuta através de um ipad, o que minimizou contato direto e risco de contaminação. Na enfermaria geral a reabilitação e o número de dias de intervenção foram maiores, entretanto na UTI, o número de sessões diárias e a duração de cada sessão foi significativamente maior.
Estabelecer se as evidências de intervenções de reabilitação testadas em populações de pacientes críticos internados em UTI (Goodwin, et al., com doenças respiratórias 2021)
Revisão sistemática de intervenções de reabilitação em pacientes com problemas respiratórios graves como a SARS, que necessitaram de cuidados intensivos, onde há sintomas paralelos com a COVID-19. Foram inclusas 24 revisões sistemáticas, 11 estudos randomizados e 8 estudos qualitativos.
Pacientes com doenças respiratórias graves e populações mistas de doenças respiratórias e condições cirúrgicas admitidos em cuidados críticos podem se beneficiar de exercícios progressivos e mobilização precoce para melhora da independência. Os estudos incluíram pacientes com doenças respiratórias graves e intervenções focadas nas deficiências cardiorrespiratórios e musculoesqueléticas que também foi observado em pessoas com COVID-19. 
Analisar a prática da Estudo de campo mobilização precoce por quantitativo e transversal fisioterapeutas intensivistas realizado com 68 em pacientes internados em fisioterapeutas intensivistas (Paulo, et al., em pacientes internados em de três hospitais da cidade 2021) UTI, as principais intervenções realizadas e as principais barreiras que impossibilitam a prática da terapia. de Fortaleza. A coleta de dados se deu por meio de formulário eletrônico através do aplicativo google forms.
$\mathrm{O}$ estudo mostrou que a sedestação, ciclo ergômetro, e a transferência leito/poltrona são as intervenções mais utilizadas pelos fisioterapeutas, sendo a escala MRC o recurso mais utilizado para avaliação. As barreiras mais prevalentes para a efetivação da mobilização precoce foram a presença de drogas sedativas/analgésicas e instabilidade hemodinâmica dos pacientes.

\begin{tabular}{|c|c|c|c|}
\hline $\begin{array}{l}\text { (Fontela, et al., } \\
\text { 2018) }\end{array}$ & $\begin{array}{l}\text { Avaliar o conhecimento } \\
\text { dos profissionais da equipe } \\
\text { multiprofissional } \\
\text { sobre mobilização precoce em } \\
\text { pacientes graves adultos, e } \\
\text { identificar atitudes e barreiras } \\
\text { percebidas para sua } \\
\text { realização. }\end{array}$ & $\begin{array}{lrr}\text { Estudo } & \text { transversal } \\
\text { realizado por meio de } \\
\text { inquérito com médicos, } \\
\text { profissionais } & \text { de } \\
\text { enfermagem } & \mathrm{e} \\
\text { fisioterapeutas de seis } \\
\text { Unidades de Terapia } \\
\text { Intensiva de dois hospitais } \\
\text { de ensino no segundo } \\
\text { semestre de 2016. As } \\
\text { respostas foram indicadas } \\
\text { utilizando uma escala } \\
\text { Likert de } 5 \text { pontos: } \\
\text { concordo totalmente, } \\
\text { concordo, neutro, discordo } \\
\text { e discordo totalmente }\end{array}$ & $\begin{array}{l}\text { A maioria dos profissionais da equipe } \\
\text { multidisciplinar afirmam obter conhecimento } \\
\text { acerca da mobilização precoce e de seus } \\
\text { benefícios, entretanto relatam como principais } \\
\text { barreiras para a sua implantação a } \\
\text { indisponibilidade de profissionais, principalmente } \\
\text { fisioterapeutas; tempo insuficiente para realizar a } \\
\text { intervenção; preocupação com autolesão } \\
\text { musculoesquelética; estresse e necessidade de } \\
\text { permanecer além de sua carga horária de trabalho. }\end{array}$ \\
\hline
\end{tabular}

Fonte: Dados da pesquisa (2021).

Os artigos mostram que a prática da mobilização precoce pode impactar diretamente na funcionalidade do paciente, contribuir para diminuição do tempo de internação e de ventilação mecânica invasiva. No entanto barreiras como sedação, instabilidade hemodinâmica e atualmente o contexto de pandemia dado pela COVID-19, tem se tornado um desafio a ser superado para a realização da mobilização precoce dentro da UTI.

A imobilidade no leito pode trazer sérias consequências físicas e psicológicas para o paciente que necessita de cuidados intensivos. Os estudos apresentados mostram a grande perda de força e massa muscular a qual o paciente acamado pode ser submetido, trazendo déficits funcionais e impacto direto na qualidade de vida do paciente pós alta hospitalar.

Um estudo prospectivo quantitativo longitudinal realizado com 33 pacientes em uma Unidade de Terapia Intensiva mostrou a relação da baixa mobilidade de pacientes com a alta taxa de mortalidade. O estudo utilizou como instrumentos de avaliação o índice proagnóstico APACHE II (Acute Physiology and Chronic Health Evaluation II) e a escala Perme Score, recentemente validada no Brasil, que considera em sua avaliação as barreiras e limitações encontradas dentro da UTI para a mobilidade de pacientes (Lima et al., 2020).

Nesse contexto, o estudo transversal de Curci et al. (2020) realizado com 32 pacientes acometidos pela COVID-19 em sua fase pós aguda, admitidos em um centro de reabilitação na Itália, mostrou que apenas 14 pacientes eram capazes de deambular, e somente 6 deambulavam de forma independente. Além disso, todos os pacientes sofriam de grau 4 ou 5 na escala MRC (Medical Research council) modificada. Segundo Curci et al. (2020) esse quadro está frequentemente associado a chamada síndrome pós terapia intensiva, reforçando a necessidade de esforços da equipe multidisciplinar para evitar essa condição ainda dentro da UTI.

Por outro lado, McWilliams et al. (2021) realizou uma análise com 111 pacientes internados em UTI acometidos pela COVID-19, embora todos esses pacientes apresentassem inicialmente fraqueza muscular adquirida na UTI, todos eles também foram mobilizados durante o tempo de internação. Dessa amostra, 50\% tiveram alta da UTI sendo capazes de levantar-se de 
uma cadeira ou andar, e ainda 55\% desses pacientes tiveram alta hospitalar sem necessitarem de mais reabilitação, mostrando assim o impacto que a mobilização precoce pode ter na alta dos pacientes.

Com a escassez de estudos voltados para a COVID-19 devido a sua incidência recente, a ASSOBRAFIR (Associação Brasileira de Fisioterapia Cardiorrespiratória e Fisioterapia em Terapia Intensiva) publicou um documento oficial afirmando que as técnicas de mobilização precoce são benéficas e necessárias para a maioria dos pacientes sob cuidados intensivos, e que não devem ser postergadas em pacientes com COVID-19 que são expostos igualmente a todos os fatores de risco comuns aos pacientes críticos (Martinez \& Andrade, 2020).

Uma revisão sistemática de Goodwin et al. (2021) reuniu as evidências atuais para reabilitação física realizada em pacientes adultos internados em UTI. Os participantes incluídos nos estudos foram alvos de intervenções de reabilitação focadas nas disfunções cardiorrespiratórias e musculoesqueléticas, que também podem ser observadas em pacientes acometidos pela COVID-19 que necessitaram de cuidados intensivos. Devido a escassez de estudos voltados para a COVID19, as intervenções abordadas nos estudos analisados por Goodwin et al. (2021), podem ser aplicadas em pacientes acometidos pela COVID-19 com o objetivo de tratar as sequelas em comum.

Além de proporcionar impacto positivo na funcionalidade do paciente, a mobilização precoce segundo o estudo de Costa et al. (2019), também é eficiente para a redução do tempo de ventilação mecânica invasiva e tempo de internação na UTI, onde foi observado uma diminuição de 2,25 dias de suporte ventilatório no grupo controle. Vale ressaltar que para a realização da mobilização precoce, se faz necessário uma avaliação individual do paciente que considere sua história atual, suas comorbidades, diagnóstico fisioterapêutico além de considerar os critérios de inclusão de pacientes elegíveis para a intervenção (Martinez \& Andrade, 2020).

Pensando nisso, Silva et al. (2021) propôs a criação de um Ckecklist Avaliativo para auxiliar os fisioterapeutas na implementação de protocolos de mobilização precoce. O instrumento criado aborda variáveis fisiológicas, clínicas, hemodinâmicas e físicas apresentadas pelos pacientes avaliados, empregando uma pontuação final que determina o grau e a intensidade da intervenção a qual esses pacientes podem ser submetidos, considerando todos os critérios de segurança. Além de promover uma avaliação pré mobilização precoce, o Checklist Avaliativo também sugere as atividades e exercícios que podem ser abordados no paciente, de maneira gradativa de acordo com a sua nota final na avaliação.

Silveira et al. (2019) em uma revisão sistemática dá ênfase a importância de uma avaliação pré mobilização precoce, para que através dela seja possível obter os potenciais benefícios da intervenção. Dentre os recursos mais utilizados nos protocolos de mobilização precoce está o cicloergômetro encontrado em mais de 50\% dos estudos analisados, que promove um aumento da força muscular periférica avaliada pela escala MRC. Em conjunto ao cicloergômetro, as técnicas de fisioterapia tradicionais que englobam exercícios passivos, treinamento a beira leito, exercícios respiratórios e treino de marcha são encontradas em todos os estudos, mostrando benefícios na mobilidade e independência do paciente após alta da UTI.

Ainda nesse contexto, Paulo et al. (2021) realizou um estudo transversal com 68 fisioterapeutas intensivistas de três hospitais diferentes na cidade de Fortaleza/CE, acerca das principais técnicas de intervenções e barreiras encontradas para a realização da mobilização precoce. Cerca de $83,8 \%$ dos fisioterapeutas afirmaram utilizar a escala MRC para a avaliação de força muscular periférica, e cerca de 91,2\% utilizam a sedestação como principal intervenção, seguido da utilização do cicloergômetro e transferências de leito para poltrona. Ainda nesse estudo, a barreira mais citada pelos fisioterapeutas intensivistas foi a presença de instabilidade hemodinâmica e presença de drogas sedativas e analgesias.

Em contrapartida, o estudo de Fontela et al. (2018) realizado com profissionais atuantes em UTI em dois hospitais de ensino, mostrou que as barreiras para a mobilização precoce vão além daquelas ligadas diretamente ao paciente. Barreiras estruturais, relacionadas também a cultura e falta de coordenação e prioridade das técnicas de mobilização precoce nas UTIs, tem sido relatada pelos profissionais. 
Apesar de os médicos, fisioterapeutas e enfermeiros reconhecerem os benefícios da mobilização precoce quanto ao ganho/manutenção de força muscular periférica, e tempo de ventilação mecânica, segundo Fontela et al. (2018) as barreiras comuns reconhecidas pelos médicos, enfermeiros e fisioterapeutas intensivistas é a indisponibilidade de profissionais na equipe, e tempo insuficiente para realização das técnicas. Além disso, fadiga, necessidade de ultrapassar carga horária de trabalho, sobrecarga e risco de autolesão também foram mencionadas pelos profissionais.

Além de todas as barreiras já citadas, McWilliams et al. (2021) dá ênfase a situação de pandemia vivenciada no mundo inteiro como mais uma barreira para a intervenção. A COVID-19 trouxe uma alta demanda de pacientes, dificultando o layout das UTIs, aumentando o risco de contaminação e levando os profissionais a priorizarem a sobrevivência, estabilidade e alta precoce para conseguirem atender a rotatividade de pacientes. Com isso, a falta de priorização da reabilitação ainda dentro da UTI tem sido evidenciada no período de pandemia, levando pacientes a alta hospitalar com grandes déficits funcionais.

Uma alternativa para a implementação da reabilitação precoce no atual contexto de pandemia, foi proposta pelo estudo de Sakai et al. (2021). Em um hospital de cuidados intensivos em Tóquio, foi formado uma equipe de reabilitação para pacientes com COVID-19 e implementado um sistema de reabilitação remota para pacientes que se encontravam na enfermaria. As enfermarias foram divididas em zona vermelha (zona infectada) e zona verde (zonas livres de contaminação), onde foi instalado terminais de Ipad conectados a Wi-fi. Dessa forma, os pacientes que atendiam aos critérios para adesão a reabilitação remota eram monitorados pelos fisioterapeutas que se encontravam na zona verde, diminuindo o risco de contaminação da equipe.

No mesmo estudo de Sakai et al. (2021) também foi realizada a reabilitação precoce em pacientes internados em UTI. Nessa condição, era exigido a paramentação completa de EPIs por parte dos profissionais, exigindo um esforço maior dos mesmos e sendo necessário um período maior de reabilitação. Em ambos os casos, a ideia de criação de uma equipe exclusiva para a realização de mobilização precoce também é defendida por Fontela et al. (2018) em seu estudo, como alternativa para a exclusão de barreiras que impendem a implementação das técnicas.

\section{Conclusão}

Nessa perspectiva, a mobilização precoce é um recurso da fisioterapia que ajuda a diminuir as sequelas deletérias do tratamento em Unidade de Terapia Intensiva, podendo diminuir o tempo de internação e de ventilação mecânica e possibilitar ao paciente uma alta hospitalar mais próxima do seu estado físico na admissão. Contudo, apesar das evidências encontradas a respeito dos benefícios da mobilização precoce, ainda existem uma série de barreiras que impossibilitam a realização eficiente da intervenção, limitações não somente ligadas diretamente ao paciente, mas também relacionadas a estrutura, organização e a equipe atuante na Unidade de Terapia Intensiva. Diante do contexto de pandemia, a alta demanda de pacientes e o evidente risco de contaminação podem ter postergado a aplicação de mobilização precoce em pacientes acometidos pela COVID-19, sendo priorizado pelas equipes intensivistas o manejo ventilatório e alta precoce da UTI, para que fosse possível atender toda a demanda de pacientes que necessitassem de cuidados intensivos. A mudança de cultura nas UTIs e a implementação de equipes exclusivas para esse tipo de abordagem é uma alternativa indicada pelas pesquisas. São necessárias mais pesquisas a respeito do tema abordado, principalmente relacionado aos pacientes acometidos pela COVID-19, para que os resultados da mobilização precoce nesses pacientes sejam mais evidenciados.

\section{Agradecimentos}

Quero agradecer primeiramente a Deus por meio de quem posso realizar todas as coisas, que toda honra e glória seja dada à Ele, o dono de toda sabedoria e conhecimento. Agradeço também a minha família que sempre me apoiou em toda a minha trajetória acadêmica, em especial minha mãe Rosiane Andrade e meus avós Carlos e Corina, sem eles nada disso seria 
possível. Agradeço ao meu noivo e futuro esposo Wesley Santos por me incentivar a não desistir, apoiar os meus sonhos e estar comigo em todos os momentos. Agradeço também a universidade e a todo o corpo docente, em especial professora Natália Gonçalves uma verdadeira inspiração de profissional fisioterapeuta e docente, grata a todo apoio, conhecimento e carinho compartilhado ao longo desses 5 anos, e a professora orientadora Bárbara Bahia que contribuiu no processo de elaboração do artigo com valiosas sugestões. Por último também agradeço a todos os meus amigos, por toda palavra de ânimo e incentivo compartilhado ao longo dessa trajetória.

\section{Referências}

Aquim, E. E., Bernardo, W. M., Buzzini, R. F., Azeredo, N. S. G., Cunha, L. S., Damasceno, M. C. P., Deucher, R. A. O., Duarte, A. C. M., Librelato, J. T., Silva, C. A. M., Nemer, S. N., Silva, S. D. F. \& Verona, C. (2019). Diretrizes Brasileiras de Mobilização Precoce em Unidade de Terapia Intensiva. Rev. Bras. Ter. Intensiva, 31(4): 434-443. https://doi.org/10.5935/0103-507X.20190084

Bonorino, K. C. \& Cani, K. C. (2020). Mobilização precoce em tempos de COVID-19. Rev. Bras. Ter. Intensiva, 32(4): 484-486. https://doi.org/10.5935/0103-507X.20200086

Cordeiro, A. L. L. \& Sarmento, G. J. V. (2020). Fisioterapia respiratória aplicada ao paciente crítico: manual prático. Editora Manole. https://manole.vitalsource.com/books/9786555762372

Costa, C. C., Leite, B. S., Fortino, C. K. \& Bastos, V. G. (2019). Avaliação de um protocolo de mobilização precoce em uma Unidade de Terapia Intensiva. Revista Conhecimento Online, 11(3): 92 - 114. https://doi.org/10.25112/rco.v3i0.1844

Curci, C., Pisano, F., Bonacci, E., Camozzi, D. M., Ceravolo, C., Bergonzi, R., Franceschi, S., Moro, P., Guarnieri, R., Ferrillo, M., Negrini, R. \& Sire, A. (2020). Early rehabilitation in post-acute COVID-19 patients: data from an Italian COVID-19 Rehabilitation Unit and proposal of a treatment protocol European Journal of Physical and Rehabilitation, 56(5): 633-41. http://dx.org/10.23736/S1973-9087.20.06339-X

Filho, C. R. C., Vasconcelos, D. B., Cunha, W. G. N., Vieira, E. E. A. \& Nogueira, F. J. S. (2020). Efeito da mobilização precoce na alta hospitalar de pacientes sob ventilação mecânica na unidade de terapia intensiva: revisão sistemática. Revista Ciência Plural, 6(3):194-209. https://doi.org/10.21680/2446$7286.2020 v 6 n 3 I D 21250$

Fontela, P. C., Forgiarini Jr, L. A. \& Friedman, G. (2018). Atitudes clínicas e barreiras percebidas para a mobilização precoce de pacientes graves em unidades de terapia intensiva adulto. Rev Bras Ter Intensiva, 30(2): 187-194. https://doi.org/10.5935/0103-507X.20180037

Goodwin, V. A., Allan, L., Bethel, A., Cowley, A., Cross, J. L., Day, J., Drummond, A., Hall, A. J., Howard, M., Morley, N., Coon, J. T. \& Lamb, S. E. (2021). Rehabilitation to enable recovery from COVID-19: a rapid systematic review. Physiotherapy, 111: 4-22. https://doi.org/10.1016/j.physio.2021.01.007

Lima, E. A, Rodrigues G., Peixoto Jr A. A., Sena R. S., Viana S. M. N. R. \& Mont'Alverne D. G. B. (2020). Mobility and clinical outcome of patients admitted to an intensive care unit. Fisioter. Mov., 33: 1-9. http://dx.doi.org/10.1590/1980-5918.032.AO67

Martinez, B. P. \& Andrade F. M. D. (2020). COVID-19 - Mobilização Precoce na Insuficiência Respiratória Aguda - ASSOBRAFIR. https://assobrafir.com.br/covid-19-mobilizacao-precoce-na-insuficiencia-respiratoria-aguda/

McWilliams, D., Weblin, J., Hodson, J., Veenith, T. \& Whitehouse, T. (2021). Rehabilitation Levels in Patients with COVID-19 Admitted to Intensive Care. Ann Am Thorac Soc, 18(1): 122-129. http://dx.doi.org/10.1513/AnnalsATS.202005-560OC

Paulo F. V. S., Viana M. C. C., Braide A. S. G., Morais, M. C. S. \& Malveira, V. M. B. (2021). Mobilização precoce a prática do fisioterapeuta intensivista: intervenções e barreiras. Rev Pesqui Fisioter, 11(2):298-306. http://dx.doi.org/10.17267/2238-2704rpf.

Pinto, B. F., Pinto, B. F. \& Dias, E. H. F. (2018). Efeitos sistêmicos da mobilização precoce em pacientes adultos internados na unidade de terapia intensiva: revisão atualizada. Fisioterapia Brasil, 19(6): 857-865. https://doi.org/10.33233/fb.v19i6.2118

Pissolato, J. S. \& Fleck, C. S. (2018). Mobilização precoce na unidade de terapia intensiva adulta. Fisioter Bras, 19(3): 377-84.

Sakai T., Hoshino, C., Hirao, M., Yamaguchi, R., Nakahara, R. \& Okawa, A. (2021). Rehabilitation Using PPE for Patients with COVID-19. Progress in Rehabilitation Medicine, 6. http://dx.doi.org/10.2490/prm.20210013.

Sales, E. M. P., Santos, J. K. M., Barbosa, T. B. \& Santos, A. P. (2020). Fisioterapia, funcionalidade e Covid-19: Revisão integrativa. Cadernos ESP. CearáEdição Especial, 14(1): 68 - 73.

Silva, L. L. C., Lima A. M. F., Brito, A. P., Silva, A. C., Silva, E. M. O., Araújo, I. C., Costa, J. N., Lima, L. C., Lira, M. D. C., Andrade, R. R. S., Costa, T. K. Q. \& Daniel, C. R. (2020). A atuação da fisioterapia no COVID-19. COVID-19 no Brasil Os Múltiplos Olhares da Ciência para Compreensão e Formas de Enfrentamento 4, (14): 128 - 134.

Silva, R. L. A., Felix, L. M. \& Moraes, F. R. (2021). Checklist de mobilização precoce. Conscientia e Saúde, 20:1-15. https://doi.org/10.5585/20.2021.19500

Silveira, A. C. C. N., Mota V. M. T., Souza F. K. V., Marçal, E., Gurgel, D. N. \& Nogueira, I. C. (2019). Análise dos recursos terapêuticos utilizados na mobilização precoce em pacientes críticos. Motricidade, 15(4): 71-80. http://dx.doi.org/10.6063/motricidade.20068

World Health Organization (2020). Coronavirus (COVID-19). https://covid19.who.int 